\section{Brillengestelle für Allergiker}

\section{Vorsicht bei Billigmodellen!}

\author{
Rund 2 Prozent aller Brillenträger in Deutschland leiden unter einer \\ Kontaktallergie. Für die Patienten Grund genug, sich mit den Mate- \\ rialien der Brillengestelle näher vertraut zu machen. Denn trotz ge- \\ setzlicher Vorschriften ist der Kontakt mit Nickel oder seinen Legie- \\ rungen wie auch mit allergieauslösenden Kunststoffen nicht ganz \\ auszuschließen. Vor allem Billigangebote sollten gemieden werden.
}

$E^{n}$ ntscheidend für die Auslösung einer allergischen Hautreaktion durch Brillenfassungen wie auch Schmuck oder Armbanduhren ist der unmittelbare Kontakt der Haut mit dem Allergen, meist einem Metall bzw. einer MetallLegierung oder einem Weichmacher. Der Körperschweiß ist sehr aggressiv und greift viele Materialoberflächen rasch an und löst die Allergene heraus. Schutzschichten - solange sie unbeschädigt sind - unterbinden diesen Prozess.

\section{Keine Sicherheit durch CE-Zeichen}

Zum einen beruhigend für den Verbraucher: Brillenfassungen unterliegen dem Lebensmittel- und Bedarfsgegenständegesetz, in denen genau festgelegt ist, welche Stoffe und Herstellungsverfahren erlaubt sind, welche Höchstmengen kritischer Stoffe abgegeben werden dürfen und wie die Produkte zu kennzeichnen sind. Richtig gefährliche Stoffe gelangen also nicht in den Handel.

Auch ist das „CE“-Zeichen für Brillenfassungen obligatorisch. Allerdings wird in den Vorschriften zur physiologischen Verträglichkeit lediglich vorgeschrieben, keine Materialien zu verwenden, „von denen bekannt ist, dass sie beim Tragen oder Anpassen bei einer signifikanten Anzahl von normal gesunden Trägern Irritationen und allergische und toxische Reaktionen bei der Berührung mit der Haut verursachen."

Da die Vergabe des CE-Zeichens in der Eigenverantwortung des Herstellers liegt, ist es auch sein Ermessen, wie er mit der Kennzeichungspflicht und dem Einsatz allergenhaltiger Werkstoffe um-

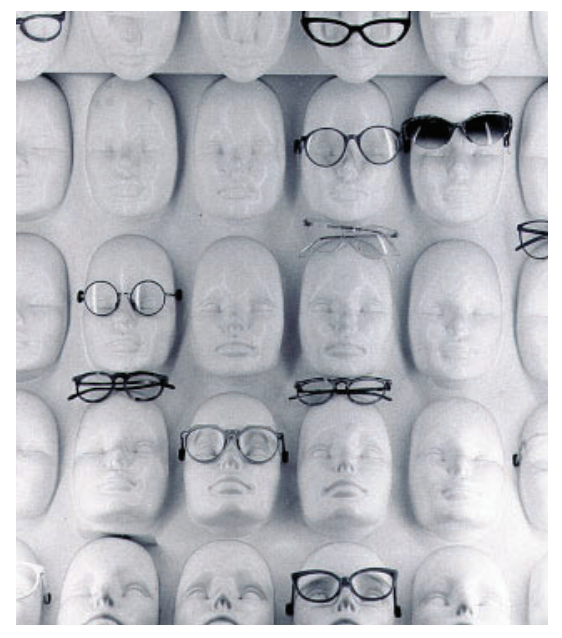

Brillenfassungen für Allergiker: Augen auf bei blankem Metall.

geht. Eine Umfrage bei marktführenden Herstellern ergab nicht immer die gewünschte Transparenz - eine der wenigen Ausnahmen war die Firma Rodenstock, so die Autoren. Vorsicht ist auf jeden Fall bei Produkten aus Billiglohnländern geboten, bei denen häufig Nickel bzw. dessen Legierungen verarbeitet und nur dünne Schutzschichten darüber gezogen werden.

\section{Schwachstelle: schlechte Passform}

Ein anderer Schwachpunkt der gesetzlichen Regelung ist der Passus „bei bestimmungsgemäßem Gebrauch...". Bestimmungsgemäßer Gebrauch bedeutet bei Brillen Berührungsstellen ausschließlich im Bereich der Bügelanlage und der Nasenauflage. Diese Bereiche müssen laut Gesetz aus nicht-allergenen Materialien gefertigt sein.
Die Erfahrung zeigt jedoch, dass viele Brillen nicht optimal sitzen und auch die Wangen oder Schläfen berühren und es hier durchaus zu allergischen Reaktionen durch nicht geeignetes Material kommen kann. Denn immer noch werden in großem Umfang nickelhaltige Legierungen für Brillenfassungen verwendet und im Bereich der Kunststoff-Fassungen sind nach wie vor weichmacherhaltige und mit UVAdsorbern ausgestattete Werkstoffe im Einsatz.

In den Bereichen, wo das Brillengestell Kontakt mit der Haut hat, werden heute Schutzschichten aus Metall oder Lack aufgetragen. Diese Schutzschichten unterliegen zwar auch bestimmten gesetzlichen Vorschriften, doch vermögen diese nicht die Eigenschaften von aggressivem Schweiß im Dauergebrauch nachzuahmen. Auch hier sollte man besonders bei Billigangeboten vorsichtig sein.

Auf der sicheren Seite ist man bei sehr teuren Fassungen aus Rein-Titan und Titan-Legierungen, ein Metall, das nach heutigem Kenntnisstand keine Hautreaktionen auslöst.

\section{Empfehlungen}

Brillenfassungen sollten so ausgesucht werden, dass ein Kontakt mit dem für den Allergiker jeweils relevanten Kontaktallergen unterbleibt. Dies ist nur bei Kenntnis aller Inhaltsstoffe möglich. Der Allergiker sollte den Augenoptiker bitten, sich auf diejenigen Fassungslieferanten zu konzentrieren, die hinsichtlich der Allergierelevanz ihrer Produkte zu eindeutigen und nachvollziehbaren nicht zu allgemein gehaltenen - Aussagen bereit sind.

Brillengestelle aus Metall sind für Nickelallergiker nicht zu empfehlen, es sei denn, es handelt sich um Titan oder das Metall ist vollständig ummantelt. Bei Kunststoffallergikern ist auf Kunststoffteile ganz zu verzichten, nur Silikon ist in diesen Fällen als Werkstoff für Nasen- und Bügelauflagen geeignet.

$b k$

\section{Kümmel D, Dormann R}

Brillen \& Co. Allergie konkret 4/99, 4-7. 